\title{
Improved General Self Cuckoo Search based Routing Protocol for Wireless Sensor Networks
}

\author{
Rubina Sharma \\ Research Scholar \\ C.S.E Department \\ GIMET, Amritsar, India
}

\author{
Richa Sharma \\ Assistant Professor \\ C.S.E Department \\ GIMET, Amritsar, India
}

\begin{abstract}
WSNs have become major area of research in computational theory due to its wide range of applications. But due to limited battery power the energy consumption has become major limitations of WSNs protocols. Though many protocols have been proposed so far to improve the energy efficiency further but still much enhancement can be done. In order to overcome the constraints of the earlier work a new improved technique is proposed in this paper. The proposed technique has the ability to overcome the limitations of the tree based routing protocol by using the reactivity and cuckoo search based optimization based optimized path selection. The proposed technique has been designed and implemented in the MATLAB tool. Various metrics have also been used to evaluate the improvement of the proposed technique over tree based routing protocol which proves the efficiency of the proposed algorithm.
\end{abstract}

\section{Keywords}

WSNs, cuckoo search, GSTEB

\section{INTRODUCTION}

A Wireless Sensor Network (WSN) [1] includes huge number of tiny sensor nodes; each node has a radio transceiver, a microprocessor and a sensor. Such sensor nodes are able to form a network on their own, sensed information is transmitted through this network. Each node has processing capacity; data is processed as it passes through network. The emerging field of wireless sensor networks combines sensing, computation, and communication into a single tiny device. The power of wireless sensor networks lies in the ability to deploy large numbers of small nodes that assemble and configure themselves. The significance of WSN is due to their ability for complete monitoring in remote and unreachable locations where it is not practical to set up conventional wired infrastructure. WSNs can revolutionize information and communication technology. Sensor nodes have restricted battery power and replacement of batteries is not an easy option because of deployment of sensor nodes over a large area so given these energy restrictions of the nodes and levels of high demands with which the nodes must operate, algorithms and protocols must be designed to provide strong and efficient energy consumption. Various protocols such as BEENINSH [2], EAP [3] and GSTEB [4] have been proposed to increase lifetime of WSNs. In spite of huge no. of protocols developed by various authors still there is room for much enhancement. In this paper, we propose a new improved protocol based on tree based routing which uses reactivity and cuckoo search optimization to improve energy efficiency.

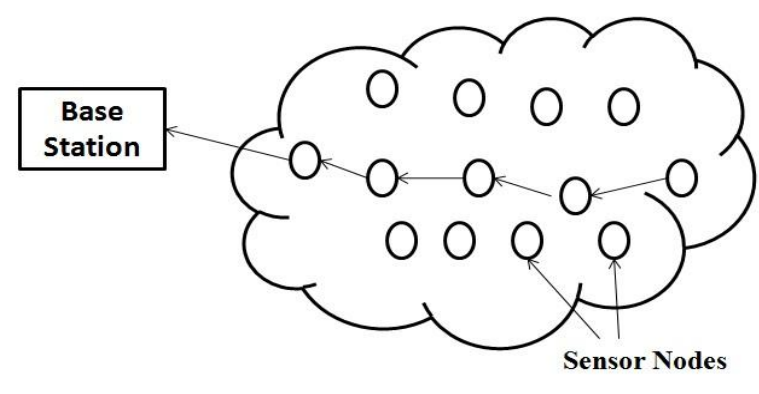

Fig 1: A wireless sensor network

\section{CUCKOO SEARCH OPTIMIZATION}

Cuckoo search (CS) optimization [5] algorithm was proposed by Xin-she Yang and Suash Deb. CS optimization was motivated from the way of reproduction adopted by some varieties of cuckoo bird, obligate brood parasitism of some cuckoo species is the main inspiration behind development of this method. These species reproduce by laying their eggs in the host birds' nest. Some host birds pose a challenge for such reproductive technique of cuckoo birds, such as, if a host bird identifies cuckoo eggs residing in its nest it will either toss away those alien eggs or desert its nest and make a new nest somewhere else. Cuckoo search is based on such reproduction behavior, and is useful for various optimization problems. It performs better than other metaheuristic algorithms.

Cuckoo search (CS) employs the following representations:

Every egg in a nest symbolizes a solution, and a cuckoo egg stands for a new solution. CS Optimization aims to use the new and potentially better solutions (cuckoos) to replace a not-so-good solution in the nests. In the simplest structure, every nest has a single egg. The algorithm can be extended to more complicated cases in which every nest has multiple eggs representing a set of solutions.

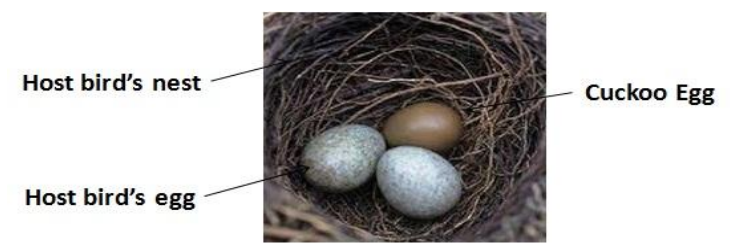

Host bird's egg $\Rightarrow$ A solution

Cuckooegg $\Rightarrow$ A new better solution

Fig 2: Representation of cuckoo search 


\section{LITERATURE SURVEY}

Smaragdakis et al. [2004] [6] proposed a heterogeneousaware protocol to increase stability period of networks. In SEP a node selects itself as cluster head based on its initial energy relative to that of other nodes. Results in the paper show that SEP always increases stability period as compared to previous clustering protocols. Qingxin Zhu et. al. [2006] [7] have proposed a new energy efficient clustering protocol named DEEC. DEEC elects cluster heads based in the residual energy of nodes. DEEC gives good performance in multilevel heterogeneous WSNs. Ming liu et al. [2007] [3] proposed a novel hierarchical clustering protocol for increasing network lifetime of sensor networks. EAP performs fairly better and increases network lifetime by reducing energy utilization and load balancing within each node. Results in the paper show that proposed protocol performs better than other protocols such as LEACH and HEED. Weifa liang et al. [2007] [8] considered an online data gathering problem in sensor networks. It is assumed that there is a series of data gathering queries arriving one by one. The system constructs a routing tree for responding to each arriving query. In the tree the volume of the data transmitted by each internal node depends on the volume of data sensed by the node and also the volume of data received from its children. The objective is to increase the number of data gathering queries answered before the first node dies. The experimental results showed that algorithm MNL performs far better than the other algorithms proposed such as MDST, MMRE, SPT, and BT. Wei ye et al. [2002] [9] proposed S-MAC, a medium-access control (MAC) protocol designed for wireless sensor networks. MAC is dissimilar from conventional wireless MACs such as IEEE 802.11 in many ways such as energy preservation and selfconfiguration are prime concerns, while per node fairness and latency are less significant. Motivated by PAMAS, S-MAC sets the radio to sleep mode while transmissions of other nodes. Unlike PAMAS, it only uses in-channel signaling. SMAC lowers contention latency for various WSN applications which need store-and-forward processing as data is passed along the network. The experiment results show that S-MAC performs better than traditional MAC. Parul saini et. al. [2010] [10] have proposed EDEEC for three types of nodes to increase network lifetime and stability. Results in the paper show that EDEEC performs better as compared to SEP. Basma M. Mohammad El-Basioni et al. [2011] [11] studied a hierarchical clustering routing protocol introduced for data assembling software in WSN called Energy-Aware routing Protocol (EAP) which fulfills various significant necessities for a clustering algorithm. EAP has various advantages, it is proven that it increases network lifetime significantly. Authors evaluate EAP in connection with network lifetime, end to end delay, packet loss percentage, and throughput, and introduce an improved protocol that performs better. The modified protocol has improved characteristics of packets loss, delay, and throughput, but decreases lifetime a little. It is shown in the paper that the modified protocol performs better than EAP. M.J. Shamani et al. [2013] [12] studies heterogeneous multi-domain WSNs. These are the systems in which different networks are members of separate domains and sensor nodes are installed at the identical physical location with heterogeneous topology. It seems that, domains life time can be maximized by mutual aid in packet forwarding; but selfishness is unavoidable from logical viewpoint. Paper explores this problem and propose an adaptive energy aware strategy which is a novel algorithm based on TIT-FOR-TAT, begins with generosity and adopts conservative manners at last. . The proposed approach put into effect cooperation among networks and minimizes the generosity slowly past each round. Results show that this algorithm can increase its network lifetime as compared with other. T.N. Qureshi et al. [2013] [2] studied that Clustering is an efficient method to improve energy efficiency. Heterogeneous WSNs have vast range of energy levels. After studying energy utilization by the clusters and large number of energy levels in heterogeneous WSN, BEENISH (Balanced Energy Efficient Network Integrated Super Heterogeneous) Protocol is introduced. It considers a network with nodes at four different levels of energy. In this protocol Cluster Heads (CHs) are selected by considering residual energy of nodes. Results in the paper prove that it is better than previously developed clustering protocols in heterogeneous WSNs. BEENISH uses the similar perception to DEEC, regarding selection of $\mathrm{CH}$. Main difference between the two protocols is that, DEEC has two types of nodes; normal nodes and advance nodes whereas BEENISH is based upon four kind of nodes; normal, advance, super and ultra-super nodes. $\mathrm{CH}$ is selected according to residual and average energy of the system. Hence, the nodes with higher residual energy have greater probability to become cluster heads than that with the low residual energy. In the paper BEENISH is proved more efficient than other protocols such as DEED, DDEEC and EDEEC for all types of WSNs in regard to stability period, network lifetime and throughput.

\section{PROPOSED ALGORITHM}

Figure 3 represents the flowchart of the proposed algorithm.

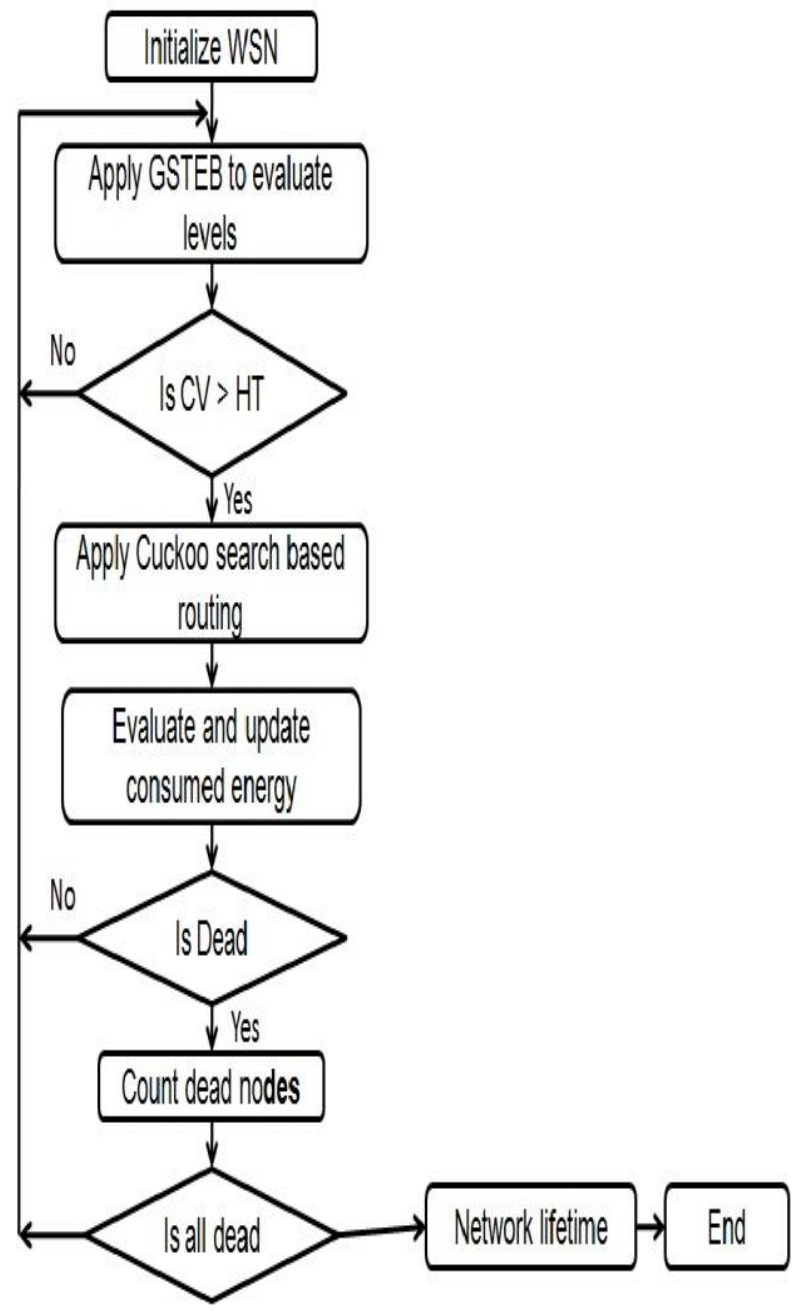

Fig 3: Flowchart of the proposed protocol

These are the steps followed to achieve proposed protocol. 
Step 1: Initialize network

Step 2: Deploy network randomly in predefined sensor field.

Step 3: Apply GSTEB to evaluate levels.

Step 4: Check if Current value (CV) > Hard threshold (HT)

Step 5: Apply cuckoo search on clusters to find the best route among nodes to sink.

Step 6: Evaluate and update energy consumption.

Step 7: Check whether all nodes become dead, if yes then show network life time and Return else continue to step 3.

\section{RESULTS AND DISCUSSIONS}

To propose reactivity and cuckoo search and tree based routing protocol for wireless sensor networks.

\subsection{Result Analysis of GSTEB}

A MATLAB simulation of GSTEB is done. We generate a randomly distributed network of 100 nodes in square area $100 \mathrm{~m} * 100 \mathrm{~m}$ with BS located at $(50 \mathrm{~m}, 190 \mathrm{~m})$.

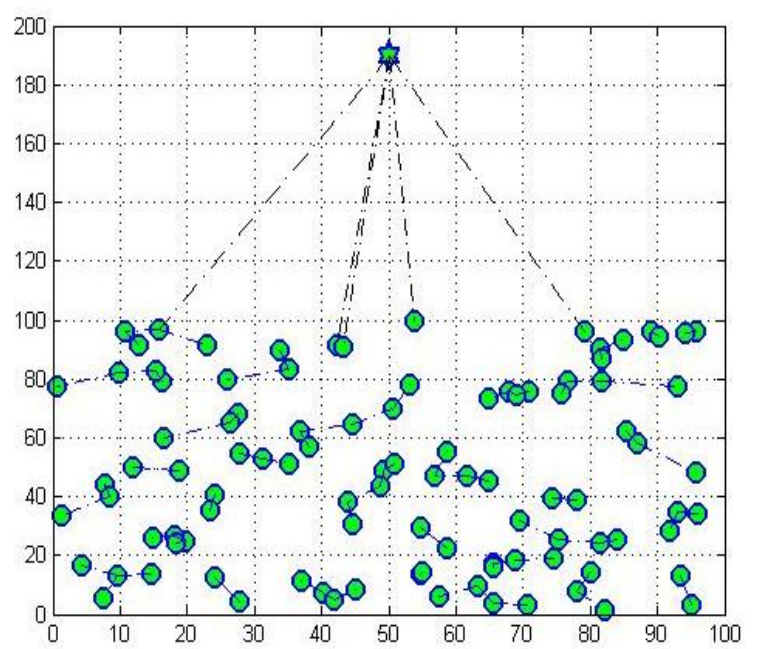

Fig 4: Routing tree constructed by GSTEB

The above figure shows the nodes in a sensor network. Green circles are normal nodes and the star is the base station to which all the nodes will send the data

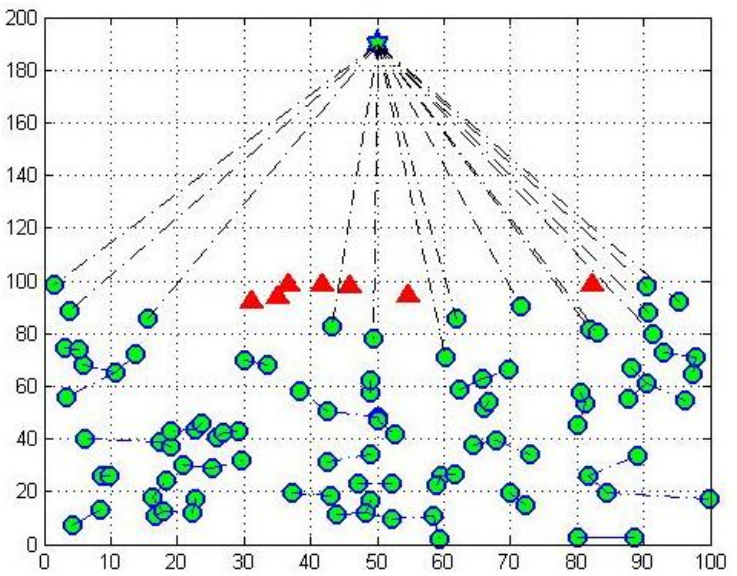

Fig 5: Nodes in Working
The above snapshot shows the working of the WSN. In working condition, the nodes send the data to the base station which is represented by the star. When whole energy of the nodes is used up in transmission and receiving of the packets then the node dies. Green circles are the working nodes and red triangles are the dead nodes.

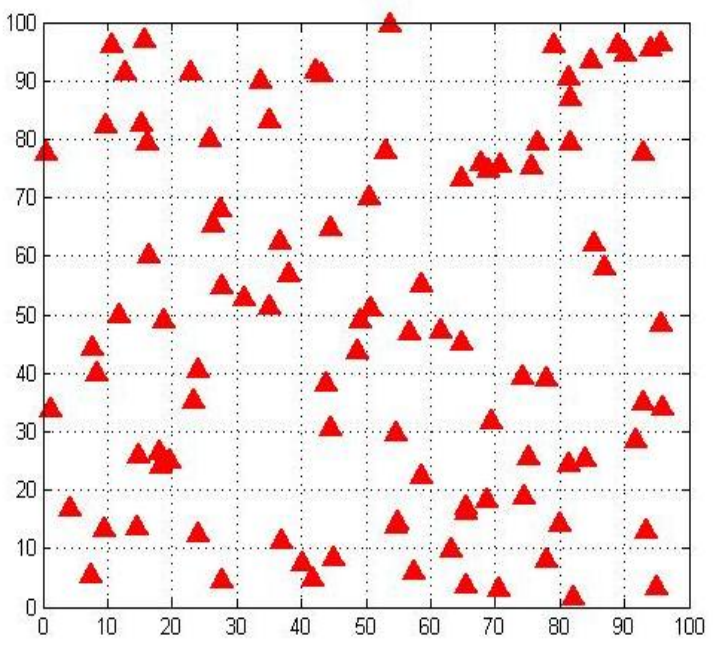

Fig 6: All Nodes Dead

In this snapshot all the nodes of the WSN are dead after transmission of the data to the base station. Red triangles represent the dead nodes.

\subsection{Result Analysis of the Proposed Protocol}

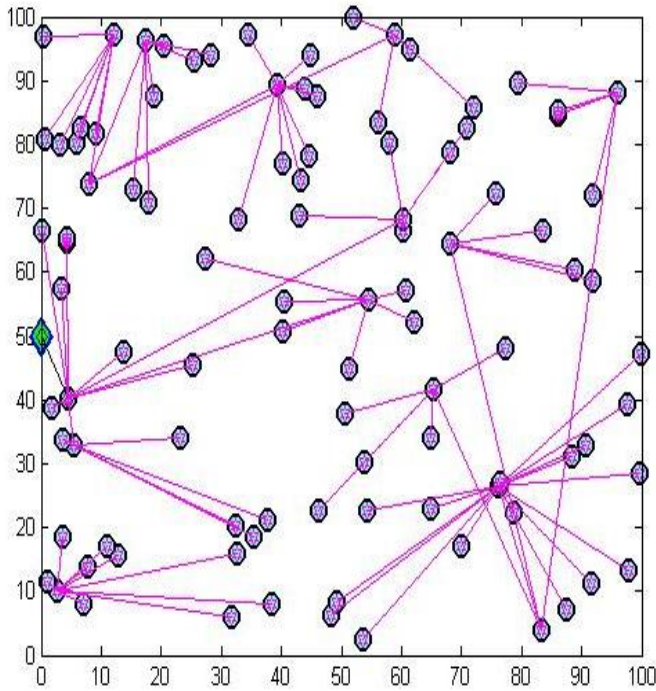

Fig 7: When all the sensor nodes are alive

Fig 7 is showing the active environment of proposed protocol. Green diamond is representing the base station. Cyan circle nodes are representing the normal nodes. Pink lines represent the communication between nodes and base station. 


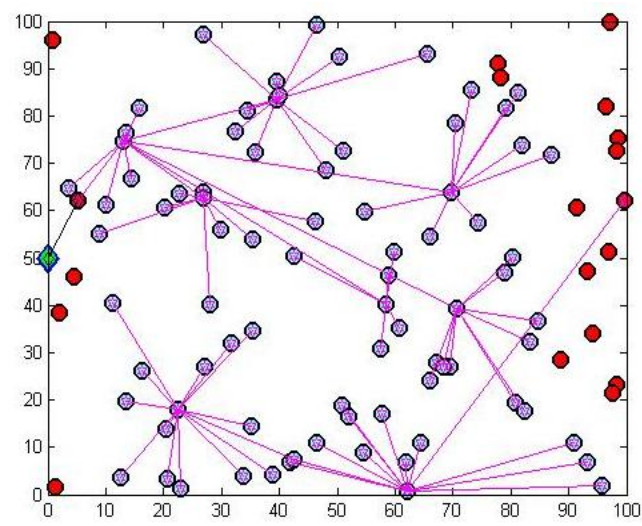

Fig 8: When some of the nodes are dead

Fig 8 is showing the network environment in which some of the nodes are dead. Dead nodes are represented by red circles.

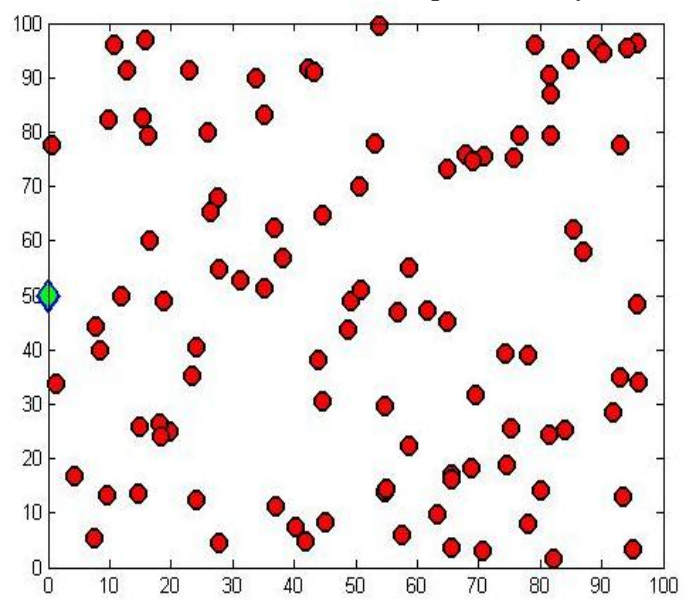

Fig 9: All nodes dead

Fig 9 shows the network when all the nodes of the WSN are dead after transmission of the data to the base station. Red circles represent the dead nodes.

In order to evaluate the effectiveness of the proposed protocol for dense networks MATLAB has been used.

FIRST NODE DEAD: - Table 1 shows the first node dead evaluation of the GSTEB and the proposed protocol. In the table, it is clearly shown that the proposed protocol performs better as compared to the GSTEB.

Table 1. First node dead evaluation

\begin{tabular}{|c|c|c|}
\hline NO. OF NODES & GSTEB & PROPOSED PROTOCOL \\
\hline 100 & 138 & 518 \\
\hline 120 & 110 & 553 \\
\hline 140 & 93 & 532 \\
\hline 160 & 72 & 549 \\
\hline 180 & 158 & 608 \\
\hline 200 & 136 & 570 \\
\hline 220 & 121 & 541 \\
\hline 240 & 108 & 479 \\
\hline 260 & 97 & 522 \\
\hline 280 & 88 & 562 \\
\hline 300 & 78 & 517 \\
\hline
\end{tabular}

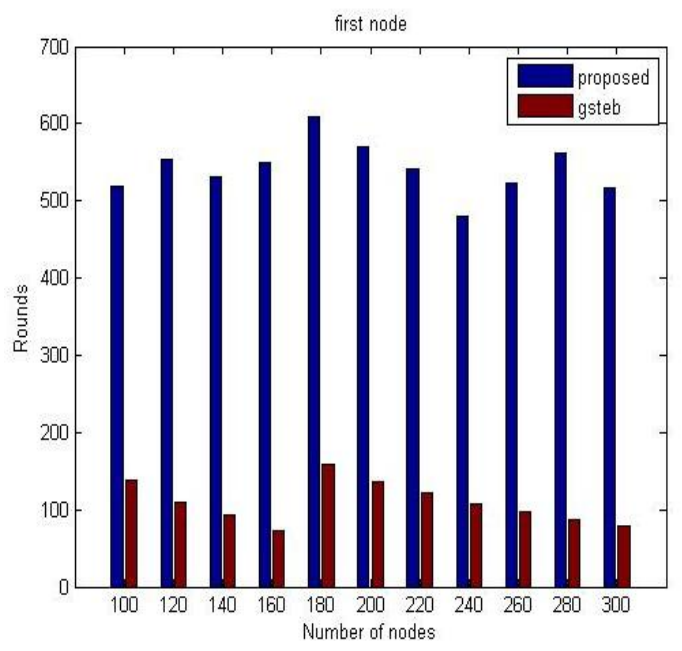

Fig 10: First node dead analysis

Figure 10 is showing the comparison of proposed protocol and GSTEB with respect to total number of rounds in case of first dead node when the number of nodes is changed. $\mathrm{X}$-axis is representing number of nodes. Y-axis is representing the number of rounds. It has been clearly shown that the overall number of rounds in case of proposed protocol are quite more than that of the GSTEB. Thus proposed protocol outperforms over GSTEB.

HALF NODE DEAD: - Table 2 shows the half node dead evaluation of the proposed protocol and the GSTEB protocols. In the table, it is clearly shown that the proposed protocol performs better as compared to GSTEB.

Table 2. Half nodes dead evaluation

\begin{tabular}{|c|c|c|}
\hline NO. OF NODES & GSTEB & PROPOSED PROTOCOL \\
\hline 100 & 294 & 684 \\
\hline 120 & 249 & 692 \\
\hline 140 & 246 & 706 \\
\hline 160 & 200 & 734 \\
\hline 180 & 284 & 734 \\
\hline 200 & 273 & 723 \\
\hline 220 & 233 & 735 \\
\hline 240 & 217 & 747 \\
\hline 260 & 202 & 721 \\
\hline 280 & 204 & 731 \\
\hline 300 & 191 & 726 \\
\hline
\end{tabular}

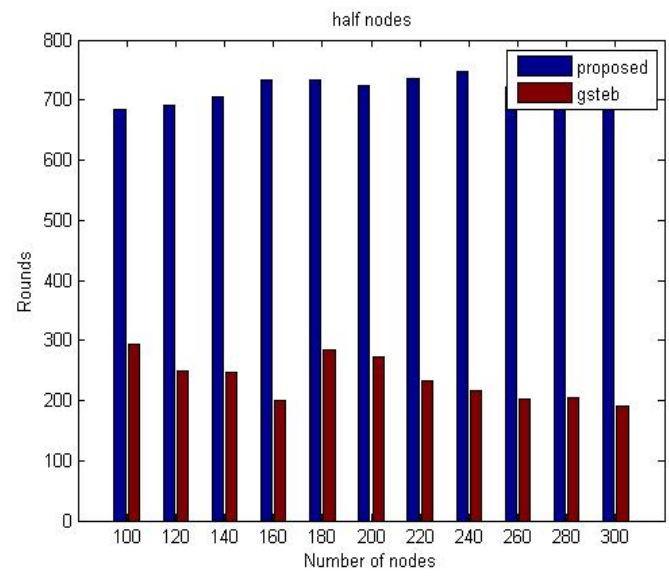

Fig 11: Half node dead analysis 
Fig 11 is showing the comparison of proposed protocol and the GSTEB with respect to total number of rounds in case of half dead node when the number of nodes are changed. $X$-axis is representing number of nodes. Y-axis is representing the number of rounds.

ALL NODES DEAD: - Table 3 shows the all node dead evaluation of the proposed protocol and the GSTEB. In the table, it is clearly shown that the proposed protocol performs better as compared to the GSTEB.

Table 3. All nodes dead evaluation

\begin{tabular}{|c|c|c|}
\hline NO. OF NODES & GATEB & PROPOSED PROTOCOL \\
\hline 100 & 329 & 719 \\
\hline 120 & 278 & 711 \\
\hline 140 & 266 & 720 \\
\hline 160 & 239 & 753 \\
\hline 180 & 307 & 757 \\
\hline 200 & 293 & 738 \\
\hline 220 & 271 & 749 \\
\hline 240 & 257 & 760 \\
\hline 260 & 246 & 741 \\
\hline 280 & 245 & 744 \\
\hline 300 & 224 & 739 \\
\hline
\end{tabular}



Fig 12 All node dead analysis

Fig 12 is showing the comparison of proposed protocol and the GSTEB with respect to total number of rounds in case of last dead node when the number of nodes are changed. $\mathrm{X}$-axis is representing number of nodes. Y-axis is representing the number of rounds. It has been clearly shown that the overall number of rounds in case of proposed protocol are quite more than that of the GSTEB. Thus our proposed protocol outperforms over the GSTEB.

\section{CONCLUSION}

In this paper, a reactivity and cuckoo search tree based routing protocol for wireless sensor networks has been proposed in order to overcome the constraint of the earlier work. Moreover the effectiveness of the proposed protocol for dense networks has been evaluated. The proposed algorithm has been designed and implemented in MATLAB. Also the comparison of the proposed technique has been done with the existing technique which proves the effectiveness of the proposed algorithm.

\section{REFERENCES}

[1] Akyildiz, I. F., Su, W., Sankarasubramaniam, Y., \& Cayirci, E. (2002). Wireless Sensor Networks: A Survey. Computer networks, 38(4), 393-422.

[2] Qureshi, T. N., et al. "BEENISH: Balanced Energy Efficient Network Integrated Super Heterogeneous Protocol for Wireless Sensor Networks." Procedia Computer Science 19 (2013): 920-925.

[3] Mohammad El-Basioni, B. M., Abd El-kader, S. M., Eissa, H. S., \& Zahra, M. M. (2011). An optimized energy-aware routing protocol for wireless sensor network. Egyptian Informatics Journal, 12(2), 61-72.

[4] Han, Z., Wu, J., Zhang, J., Liu, L., \& Tian, K. (2014). A general self-organized tree-based energy-balance routing protocol for wireless sensor network.Nuclear Science, IEEE Transactions on, 61(2), 732-740.

[5] “Cuckoo Search", [Last Visited] 5.May.2015, [ONLINE AVAILABLE] http://en.wikipedia.org/wiki/Cuckoo_search.

[6] Smaragdakis, Georgios, Ibrahim Matta, and Azer Bestavros. "SEP: A stable election protocol for clustered heterogeneous wireless sensor networks." InSecond international workshop on sensor and actor network protocols and applications (SANPA 2004), pp. 1-11. 2004.

[7] Qing, Li, Qingxin Zhu, and Mingwen Wang. "Design of a distributed energy-efficient clustering algorithm for heterogeneous wireless sensor networks."Computer communications 29.12 (2006): 2230-2237.

[8] Liu, M., Zheng, Y., Cao, J., Chen, G., Chen, L., \& Gong, H. (2007, June). An energy-aware protocol for data gathering applications in wireless sensor networks. In Communications, 2007. ICC'07. IEEE International Conference on(pp. 3629-3635). IEEE.

[9] Liang, Weifa, and Yuzhen Liu. "Online data gathering for maximizing network lifetime in sensor networks." Mobile Computing, IEEE Transactions on 6.1 (2007): 211 .

[10] Saini, Parul, and Ajay K. Sharma. "E-DEEC-enhanced distributed energy efficient clustering scheme for heterogeneous WSN." In Parallel Distributed and Grid Computing (PDGC), 2010 1st International Conference on, pp. 205-210. IEEE, 2010.

[11] Ye, Wei, John Heidemann, and Deborah Estrin. "An energy-efficient MAC protocol for wireless sensor networks." INFOCOM 2002. Twenty-First Annual Joint Conference of the IEEE Computer and Communications Societies. Proceedings. IEEE. Vol. 3. IEEE, 2002.

[12] Shamani, M. J., et al. "Adaptive Energy Aware Cooperation Strategy in Heterogeneous Multi-domain Sensor Networks." Procedia Computer Science 19 (2013): 1047-1052. 